\title{
Three dimensional electrospun PCL/PLA blend \\ nanofibrous scaffolds with significantly improved stem cells osteogenic differentiation and cranial bone formation
}

Qingqing Yao ${ }^{\mathrm{a}, \dagger}$, Jaqueline G.L. Cosme ${ }^{\mathrm{b}, \mathrm{c}, \dagger}$, Tao Xu ${ }^{\mathrm{b}}$, Jacob M. Miszuk ${ }^{\mathrm{a}}$, Paulo H.S. Picciani $^{\mathrm{c}}$, Hao Fong ${ }^{\mathrm{b}, * *}$, Hongli Sun ${ }^{\mathrm{a}, *}$

${ }^{a}$ Biomedical Engineering Department, University of South Dakota, Sioux Falls, SD 57107,

USA

${ }^{\mathrm{b}}$ Program of Biomedical Engineering, South Dakota School of Mines and Technology, Rapid City, SD 57701, USA

${ }^{\mathrm{c}}$ Institute of Macromolecules, Federal University of Rio de Janeiro, Rio de Janeiro, RJ 21941-901, Brazil

$\dagger$ These authors contributed equally to this work.

Corresponding Authors

* Professor Hongli Sun, Ph.D.

Phone: (+1) 605-275-7470; Fax: +1 605-782-3280; E-mail: Hongli.Sun@usd.edu ** Professor Hao Fong, Ph.D.

Phone: (+1) 605-394-1229; Fax: +1 605-394-1232; E-mail: Hao.Fong@sdsmt.edu

\section{Biomaterials}


Abstract: Nanofibrous scaffolds that are morphologically/structurally similar to natural ECM are highly interested for tissue engineering; however, the electrospinning technique has the difficulty in directly producing clinically relevant 3D nanofibrous scaffolds with desired structural properties. To address this challenge, we have developed an innovative technique of thermally induced nanofiber self-agglomeration (TISA) recently. The aim of this work was to prepare (via the TISA technique) and evaluate 3D electrospun PCL/PLA blend (mass ratio: 4/1) nanofibrous scaffolds having high porosity of $\sim 95.8 \%$ as well as interconnected and hierarchically structured pores with sizes from sub-micrometers to $\sim 300 \mu \mathrm{m}$ for bone tissue engineering. The hypothesis was that the incorporation of PLA (with higher mechanical stiffness/modulus and bioactivity) into PCL nanofibers would significantly improve human mesenchymal stem cells (hMSCs) osteogenic differentiation in vitro and bone formation in vivo. Compared to neat PCL-3D scaffolds, PCL/PLA-3D blend scaffolds had higher mechanical properties and in vitro bioactivity; as a result, they not only enhanced the cell viability of hMSCs but also promoted the osteogenic differentiation. Furthermore, our in vivo studies revealed that PCL/PLA-3D scaffolds considerably facilitated new bone formation in a critical-sized cranial bone defect mouse model. In summary, both in vitro and in vivo results indicated that novel 3D electrospun PCL/PLA blend nanofibrous scaffolds would be strongly favorable/desired for hMSCs osteogenic differentiation and cranial bone formation.

Keywords: 3D electrospun nanofibrous scaffold; Polycaprolactone; Polylactic acid; Osteogenic differentiation; Bone regeneration 


\section{Introduction}

Presently, the widely adopted approach for large bone defect repair is autogenous bone graft; however, this approach has several problems such as the limited availability of autogenous bone and the risk of donor site morbidity [1, 2]. Alternatively, allogenic bone graft can be used; nevertheless, it suffers from the concern of disease transmission and immunoreactions [3-5]. As a result, scaffold-based tissue engineering techniques have attracted growing interests $[6,7]$. A scaffold can act as an engineered three dimensional (3D) microenvironment for cell adhesion, proliferation, differentiation, and extracellular matrix (ECM) formation [8-10]. It is important to note that the microenvironment determined by both physical and chemical properties of a scaffold plays the crucial role on cell functions and the subsequent tissue regeneration.

In natural ECM (e.g., the ECM of bone tissue), collagen fibers with diameters ranging from tens to hundreds of nanometers are the main component [11]; whereas in tissue engineering, how to develop highly biomimetic nanofibrous scaffolds is still a major technological challenge. To date, several techniques including phase separation, self-assembly, and electrospinning have been investigated to prepare nanofibrous scaffolds as ECM substitutes [12-16]; among them, the electrospinning technique has been paid particular attention because of its capability to make nanofibers with morphological structures similar to those in natural ECM. Furthermore, the electrospinning technique is facile while versatile; it is able to process various polymer materials, and the resulting electrospun nanofibers (usually collected as overlaid mats) are cost-effective and favorable for a variety of cellular functions 
[16-18]. It is necessary to note that the interconnected macroporous structures are essential for cell growth and tissue regeneration; whereas as-electrospun nanofibrous mats do not possess macoroporous structures, which hinders the infiltration/growth of cells. In other words, as-electrospun nanofibrous mats generally behave similar to 2D scaffolds (e.g., films). Hence, it is important to develop innovative strategies for making biologically and clinically relevant 3D electrospun nanofibrous scaffolds with desired morphological/structural properties (e.g., interconnected macropores and high porosity) [19]. Although the phase separation technique can be combined with the porogen leaching technique (TIPS\&P) to make nanofibrous scaffolds with interconnected macropores [20, 21], some limitations significantly impede its application. For example, the phase separation technique has little control over the diameter/orientation of fibers in a scaffold, and the preparation time is typically long. Moreover, the complete removal of porogen from a resulting scaffold is often a problem/difficulty. Additionally, the available polymers for making TIPS\&P scaffolds are very limited; whereas the electrospun scaffolds can be made from a variety of polymers with well controlled fiber size and orientation, which is very important for the prepared medical devices to meet different kinds of needs.

In our recently published paper [22], a new strategy of thermally induced (nanofiber) self-agglomeration (TISA) followed by freeze drying has been reported; in specific, as-electrospun polycaprolactone (PCL) nanofibrous mats can first be converted into short individual nanofibers and tiny nanofibrous pieces, which can subsequently be utilized as building materials for making 3D electrospun PCL nanofibrous scaffolds. The resulting 3D 
scaffolds have the porosity of $\sim 96 \%$ and possess the interconnected and hierarchically structured pores including macropores with sizes up to $300 \mu \mathrm{m}$. Therefore, we believe that our TISA technique is highly innovative; and the resulting scaffolds have more advantages as compared to previously reported scaffolds (e.g., the TIPS\&P scaffolds).

Our in vitro cell differentiation results indicated that 3D electrospun PCL nanofibrous scaffolds (prepared via the TISA technique) were more favorable for chondrogenic (rather than osteogenic) differentiation, probably attributed to the relatively low stiffness/modulus of PCL [22]. Although these scaffolds could support bone morphogenetic protein 2 (BMP2) induced ectopic endochondral bone formation, new bones were identified merely in the surrounding areas but not inside the implanted scaffolds, suggesting that these scaffolds might need improvements to encourage/facilitate the bone formation. It is known that PCL is soft and elastic; although this might be advantageous for bone formation by promoting the chondrogenic differentiation through mimicking the physiological endochondral ossification, appropriate stiffness/modulus would still be required to provide adequate mechanical support for early cell growth and the subsequent tissue regeneration, particularly under the in vivo condition. Furthermore, slow degradation and bio-inertness of PCL may also impede new bone formation. Hence, it is necessary to improve the osteogenic differentiation and bone formation by judiciously tailoring the mechanical and chemical properties of $3 \mathrm{D}$ electrospun nanofibrous scaffolds.

Owing to biocompatibility, biodegradability, and relatively high mechanical properties, polylactic acid (PLA, an FDA approved biopolymer) has been widely adopted for 
biomedical applications [23]. Although both PCL and PLA are linear aliphatic polyesters, the difference on molecular compositions makes PCL a more flexible, hydrophobic, and crystalline polymer that is slower to degrade than PLA; on the other hand, PLA has higher stiffness/modulus and strength than PCL [24]. Upon mixing PLA and PCL together (i.e., upon the formation of PCL/PLA blend), the merits of both polymers could be retained; meantime, the problems could be mitigated $[25,26]$. With the incorporation of PLA into PCL, the resulting 3D electrospun PCL/PLA blend nanofibrous scaffolds (prepared via the TISA technique) would have shorter degradation time and higher mechanical properties (e.g., stiffness and modulus) than the neat PCL scaffolds; moreover, the bioactivity might be improved. Note that the PCL/PLA blends have not been as widely studied as the PCL/PLA copolymers due to the concerns over immiscibility of PCL and PLA. Nevertheless, recent research endeavors have revealed that the PCL/PLA blends could be made via the solvent evaporation method; when the majority (i.e., $70 \mathrm{wt} . \%$ or higher) of a PCL/PLA blend was PCL, it appeared that the phase separation would not be a major issue/concern $[26,27]$.

In this work, the TISA technique was explored to make 3D electrospun PCL/PLA blend nanofibrous scaffolds first; thereafter, the prepared scaffolds were studied to improve human mesenchymal stem cells (hMSCs) osteogenic differentiation in vitro and bone formation in vivo. Our hypothesis was that the incorporation of relatively stiff and bioactive PLA into the 3D scaffolds would further enhance the osteogenic differentiation of hMSCs, thus facilitating new bone formation. The in vitro hydroxyapatite formation ability on $3 \mathrm{D}$ electrospun PCL/PLA blend nanofibrous scaffolds was investigated by using a simulated 
body fluid (SBF), while the cell viability and osteogenic differentiation of hMSCs were studied upon measuring the gene expression, ALP activity, and calcium content. Moreover, a clinically relevant critical-size cranial bone defect mouse model was adopted to investigate the in vivo bone formation ability of these 3D PCL/PLA blend nanofibrous scaffolds.

\section{Experimental}

\subsection{Materials}

PLA resin 4042D (made from 95.8\% L-lactide and 4.2\% D-lactide, $\mathrm{M}_{\mathrm{w}}=66,000$ $\mathrm{g} / \mathrm{mol}$ ) was purchased from NatureWorks LLC (Minnetonka, MN). PCL $\left(\mathrm{M}_{\mathrm{w}}=80,000\right.$ $\mathrm{g} / \mathrm{mol}$ ), gelatin (G1890), ethanol, dichloromethane (DCM), N,N-dimethylformamide (DMF) were purchased from Sigma-Aldrich (St. Louis, MO). All of the chemicals/materials were used as received.

\subsection{Electrospinning}

PCL and PCL/PLA (a polymer blend with the PCL/PLA mass ratio of 4/1) solutions were prepared by dissolving each polymer in a mixture solvent of DCM/DMF (with the volume ratio of 60/40). The overall concentrations of both solutions were 8 wt.\%. Each solution was then put in a $30 \mathrm{~mL}$ BD Luer-Lok tip plastic syringe having a stainless-steel needle with 18 gauge $90^{\circ}$ blunt end. The electrospinning setup consisted of a high voltage power supply purchased from Gamma High Voltage Research (Ormond Beach, FL) and a digitally controlled syringe pump purchased from KD Scientific (Holliston, MA). As-electrospun nanofibrous mats were collected on a laboratory-produced roller (with the 
diameter of $25 \mathrm{~cm}$ ) covered with aluminum foil. During electrospinning, the distance between the tip of syringe and the surface of roller was set at $25 \mathrm{~cm}$, while the rotational speed of roller was set at $100 \mathrm{rpm}$. For PCL solution, the flow rate and applied voltage were set at 3 $\mathrm{mL} / \mathrm{h}$ and $13 \mathrm{kV}$; while for PCL/PLA blend solution, the flow rate and applied voltage were set at $1.5 \mathrm{~mL} / \mathrm{h}$ and $15 \mathrm{kV}$.

\subsection{Preparation of short individual nanofibers and tiny nanofibrous pieces}

Each as-electrospun nanofibrous mat was first cut into small pieces (with length and width of $\sim 1 \mathrm{~cm}$ ) and then soaked with ethanol; subsequently, the soaked nanofibrous mats/pieces were placed into a mortar which contained liquid nitrogen. The frozen mats/pieces were then converted into short individual nanofibers and tiny nanofibrous pieces upon mechanical grinding. During this process, liquid nitrogen was continuingly added into the mortar until the mats/pieces were thoroughly ground. Thereafter, they were dispersed in ethanol and then went through a mesh/sieve (with the pore size of $\sim 1 \mathrm{~mm}$ ) to acquire the short individual nanofibers and tiny nanofibrous pieces. Eventually, the leftover large mats/pieces were placed back into the mortar for further grinding. The above procedure was repeated for several times until almost all of short individual nanofibers and tiny nanofibrous pieces were collected. After that, they were dispersed in ethanol and then placed into a glass flask. After the system was stored under the ambient condition for $48 \mathrm{~h}$, short individual nanofibers and tiny nanofibrous pieces would precipitate at the bottom of flask; while clear ethanol at the top of flask was carefully removed by using a glass Pasteur pipette.

\subsection{Fabrication of 3D nanofibrous scaffolds}


PCL-3D and PCL/PLA-3D nanofibrous scaffolds were fabricated by using the TISA technique followed by the freeze drying method [22]. In brief, the acquired short individual nanofibers and tiny nanofibrous mats/pieces of PCL or PCL/PLA blend (that were dispersed in ethanol) were first mixed with a gelatin aqueous solution; and the volume ratio of ethanol/water/gelatin was set at 4/2/1. The obtained dispersion was then submerged into a water bath at $54{ }^{\circ} \mathrm{C}$ for $2.5 \mathrm{~min}$. During this time period, the short individual nanofibers and tiny nanofibrous pieces of PCL or PCL/PLA spontaneously turned into 3D agglomerate; immediately after that, the system was placed into ice water for 2 min to prevent the 3D agglomerate from further shrinkage/agglomeration. Thereafter, the obtained 3D agglomerate was rinsed with distilled water for 3 times to remove the residual ethanol and gelatin before the subsequent freeze drying. For making the 3D nanofibrous scaffold, the 3D agglomerate soaked with distilled water was first placed at $-15^{\circ} \mathrm{C}$ for $1 \mathrm{~h}$ to turn water into ice and then submerged in liquid nitrogen for a few minutes; finally, the sample was put into a pre-cooled glass flask followed by being freeze dried at room temperature for $24 \mathrm{~h}$.

\subsection{Control samples}

As-electrospun nanofibrous mats (denoted as PCL-2D and PCL/PLA-2D scaffolds) were also prepared and studied as control samples. The mats had the thickness of $\sim 120 \mu \mathrm{m}$ and the mass per unit area of $\sim 40 \mathrm{~g} / \mathrm{m}^{2}$, and they consisted of almost randomly overlaid nanofibers of PCL or PCL/PLA blend. During in vitro and in vivo studies of these PCL/PLA-3D scaffolds, the previously reported PCL-3D scaffolds were also studied as control samples. 


\subsection{Characterization}

A Zeiss Supra 40VP field-emission scanning electron microscope (SEM) was employed to characterize morphological structures of various samples. The thickness of as-electrospun nanofibrous mat was measured by using the CO-030025 electronic digital micrometer purchased from Marathon Watch Company (Richmond Hill, Ontario, Canada). The porosity was calculated from the following equation:

$$
P_{\text {scaffold }}=\frac{V-V_{P}}{V} \times 100 \%
$$

where $P_{\text {scaffold }}$ is the porosity of scaffold, $V$ is the total volume of scaffold, and $V_{p}$ is the volume of PCL or PCL/PLA nanofibers (i.e., the mass divided by the density $\left(1.145 \mathrm{~g} / \mathrm{cm}^{3}\right)$ of PCL and $\left(1.165 \mathrm{~g} / \mathrm{cm}^{3}\right)$ of PCL/PLA blend).

\subsection{Mechanical test}

Mechanical properties of PCL-3D and PCL/PLA-3D scaffolds were measured with the MTS insight single column $100 \mathrm{~N}$ electromechanical testing system purchased from MTS Systems Co. (Edem Prarie, MN) at a crosshead speed of $1 \mathrm{~mm} / \mathrm{min}$. The samples were cut into cylindrical shape with the diameter of $5 \mathrm{~mm}$ and the height of $3 \mathrm{~mm}$. The mechanical test of samples was carried out at both dry condition (desiccated for several days) and wet condition (immersed in phosphate-buffered saline (PBS) for at least $24 \mathrm{~h}$ ). The modulus value was determined from linear region of the acquired stress-strain curve.

\subsection{Assessment of bioactivity in SBF}

The mineralization process of PCL and PCL/PLA nanofibrous scaffolds was studied in Kokubo's SBF [28]. Each sample was soaked in $15 \mathrm{~mL}$ of SBF and placed in an incubator 
at $37{ }^{\circ} \mathrm{C}$ with constant shaking at $60 \mathrm{rpm}$ for 4,7 , and 14 days. The SBF was refreshed twice a week. At the end of each incubation time, samples were removed from SBF, washed with deionized water, frozen at $-20{ }^{\circ} \mathrm{C}$ overnight, and then freeze dried for $24 \mathrm{~h}$. The chemical compositions of scaffolds before and after SBF immersion were determined by attenuated total reflectance (ATR) spectroscopy (Nicolet, USA). The spectra were collected in transmission mode in the mid-IR range $\left(4000-400 \mathrm{~cm}^{-1}\right)$.

\subsection{In vitro cell study}

\subsubsection{Cell seeding on PCL-3D and PCL/PLA-3D nanofibrous scaffolds}

PCL-3D and PCL/PLA-3D nanofibrous scaffolds were first cut into discs $(5 \mathrm{~mm}$ diameter $\times 2 \mathrm{~mm}$ thickness) with tissue punch, the samples were then immersed in $70 \%$ ethanol for $30 \mathrm{~min}$ followed by being washed with PBS for 3 times. Subsequently, these samples were incubated in minimum essential medium $\alpha$ ( $\alpha$-MEM, Gibco, Waltham, MA) for 30 min. The residual medium on a scaffold was removed with sterile gauze before hMSCs were seeded into the scaffold $\left(1 \times 10^{5}\right.$ cells per scaffold $)$. All of the cells/scaffolds were cultured in 24 -well plate on an orbital shaker $(30 \mathrm{rpm})$ at $37{ }^{\circ} \mathrm{C}$ with $5 \% \mathrm{CO}_{2}$.

\subsubsection{Cell viability}

Cell viability was quantitatively analyzed using CellTiter $96 \mathrm{AQ}_{\mathrm{ueous}}$ One Solution Cell Proliferation Assay (MTS, Promega, USA) according to the manufacture's instruction. In brief, after culturing for 1 and 3 days, the culture medium was removed; fresh medium with $10 \%$ MTS was then added, and incubated at $37{ }^{\circ} \mathrm{C}$ with $5 \% \mathrm{CO}_{2}$ in dark for $1 \mathrm{~h}$. The 
absorbance was measured at $490 \mathrm{~nm}$ using a microplate reader (Infinite M200, Tecan, USA). The relative cell viability (\%) was expressed as percentage relative to the control group.

hMSCs morphologies on PCL-3D and PCL/PLA-3D nanofibrous scaffolds were visualized by staining with Texas red-X Phalloidin (Life technologies, OR, USA) and DAPI (Southern Biotech, Birmingham, AL), which could label F-actin and cell nuclear, respectively [22]. In specific, cell-seeded scaffolds were fixed in 3.7\% paraformaldehyde for 10 min and permeabilized with $0.1 \%$ TritonX-100 for another 5 min. Thereafter, the samples were blocked with $1 \%$ bovine serum albumin for $30 \mathrm{~min}$ before they were stained with Texas Red and DAPI for 20 and 5 min, respectively. The cells/scaffolds were examined by using a laser scanning microscope (FV1200, Olympus, Japan).

\subsubsection{ALP activity and calcium content}

ALP activity was measured by using an EnzoLyte pNPP Alkaline Phosphatase Assay Kit (AnaSpec, San Jose, CA), as we previously reported [29] with minor modifications. Briefly, cells/scaffolds were rinsed with PBS solution and lysed with lysis buffer for 1-2 min at room temperature. The lysate was then transferred into a tube and centrifuged for $15 \mathrm{~min}$ at $2500 \mathrm{~g}$ at $4{ }^{\circ} \mathrm{C}$. The collected supernatant or standard solution $(50 \mu \mathrm{L})$ was then mixed with p-nitrophenyl phosphate and incubated for $30 \mathrm{~min}$ at $37^{\circ} \mathrm{C}$. Following the incubation, the reaction was stopped by adding $100 \mu \mathrm{L}$ terminated liquid. ALP activity was measured at 405 $\mathrm{nm}$ and normalized against total protein content. The total protein content was measured with a BCA kit (Thermo Scientific ${ }^{\mathrm{TM}}$, Waltham, MA) according to the manufacture's instruction. Briefly, $25 \mu \mathrm{L}$ of the collected supernatant (the same from ALP activity) or standard solution 
was mixed with $200 \mu \mathrm{L} \mathrm{BCA}$ working reagent and incubated for $30 \mathrm{~min}$ at $37{ }^{\circ} \mathrm{C}$. Following the incubation, the protein content was measured at $562 \mathrm{~nm}$. The cell-scaffold constructs were also examined for calcium deposition by using a total calcium LiquiColor ${ }^{\circledR}$ kit (Stanbio Laboratory, TX). After 3 weeks of culture, cells/scaffolds were rinsed with DPBS and cut into small pieces with a sharp blade. The calcium was extracted by using $1 \mathrm{~mL} 6 \mathrm{M}$ hydrochloric acid. Thereafter, $10 \mu \mathrm{L}$ extraction solution or $10 \mu \mathrm{L}$ standard solution was added into $1 \mathrm{~mL}$ working solution prepared according to the manufacturer's instruction. The absorbance was measured at $550 \mathrm{~nm}$, and the calcium content was calculated from the following equation:

$$
\text { Calcium }(m g / d L)=\frac{A u}{A s} \times 10
$$

where $\mathrm{Au}$ and As are the absorbance values of sample and standard, respectively.

\subsubsection{Gene expression analysis}

Quantitative gene expression analysis was carried out as we previously reported [30] with minor modifications. In specific, total RNA was extracted using the GeneJET ${ }^{\mathrm{TM}}$ RNA Purification Kit (Thermo Scientific ${ }^{\mathrm{TM}}$, Waltham, MA) by following the manufacturer's instruction. RNA concentration was measured with UV-vis spectroscopy (DU 730, Beckman coulter) at $260 \mathrm{~nm}$, an equivalent amount of RNA was processed to generate cDNA by using the High Capacity cDNA Reverse Transcript kit purchased from Applied Biosystems (Forster City, CA). Quantitative PCR was performed with Taqman gene expression assays (Applied Biosystems, Forster City, CA) using the Applied Biosystems 7500 Fast Real-Time PCR System (Applied Biosystems, Carlsbad, CA). Triplicates were performed for each sample, 
and the results were normalized to $\beta$-actin. Taqman gene expression assays of $\beta$-actin (Hs01060665), ALP (Hs00758162), and Bone sialoprotein (BSP) (Hs00173720) were purchased from Applied Biosystems (Forster City, CA).

\subsection{Bone regeneration in vivo}

\subsubsection{Critical-sized cranial bone defect model}

Care and use of laboratory animals followed the protocol approved by the Institutional Animal Care and Use Committee (IACUC) of the University of South Dakota. Inbred C57BL/6NHsd male mice (5-6 weeks, Envigo) were used to create critical-sized cranial bone defect model for in vivo study as we previously described [31]. Briefly, the mice were shaved and prepared with an antiseptic solution (chlorhexidine) scrub. A $\sim 0.7 \mathrm{~cm}$ full-thickness incision was made and one $5 \mathrm{~mm}$ defect was created within the parietal bone with a trephine bur. The periosteum was completely cleared from the surface of the cranium by scraping while the underlying dura mater was kept intact. Sterile scaffolds $(\Phi 5 \mathrm{~mm} \times 1 \mathrm{~mm})$ were directly placed in the cranial defects and the overlying tissue was closed with surgical staples. Staples were removed 10 days after surgery. Some scaffolds were supplemented with low dose $(0.75 \mu \mathrm{g})$ of recombinant human BMP2 (rhBMP2, Peprotech, Rocky Hill, NJ, USA), which was re-suspended in $10 \mu \mathrm{L}$ of collagen I (Bedford, MA, USA) and then incorporated into PCL-3D or PCL/PLA-3D scaffolds. A total of 24 mice were used in our animal experiments, divided into 4 groups $(n=6)$ and treated as follows: (1) PCL group: PCL-3D scaffolds were implanted to these mice with critical defects. (2) PCL/PLA group: the mice were implanted with PCL/PLA-3D scaffolds. (3) PCL-rhBMP2 group: the mice were 
implanted with PCL-3D scaffolds $+0.75 \mu \mathrm{g}$ rhBMP2. (4) PCL/PLA-rhBMP2 group: the mice were implanted with PCL/PLA-3D scaffolds $+0.75 \mu \mathrm{g}$ rhBMP2. All of the mice were euthanized at 6 weeks after surgery. Retrieved samples were fixed in $10 \%$ formalin for 2 days and then moved into $70 \%$ ethanol for further analysis. Some samples were not counted at the end of the experiment because some animals died (during or after surgery), or some implants shifted out of the defects. The counted sample number for each group was: 5, 6, 5, and 6 for PCL, PCL/PLA, PCL-rhBMP2, and PCL/PLA-rhBMP2 groups, respectively.

\subsubsection{Radiographic and histological analysis}

Radiographic analysis was performed on the fixed constructs using an In-Vivo Xtreme small animal imaging system (Bruker, Billerica, MA, USA). The formalin fixed samples were decalcified with $15 \%$ EDTA $(p \mathrm{H}=7.2)$ solution for 3 days before they were embedded in paraffin for further histological analysis. Five-micrometer cross-sections were cut from the middle of scaffolds and stained with Hematoxylin and Eosin (H\&E) for microscopic observation. The percentage of new bone area of each specimen (new bone area in scaffold/total scaffold area $\times 100 \%)$ was measured by using the Image J software $(\mathrm{NIH}$, Bethesda, MD, USA).

\subsection{Statistical analysis and image editing}

To determine statistical significance of observed differences between the study groups, a two-tailed homoscedastic t-test was applied. A value of $\mathrm{p}<0.05$ was considered to be statistically significant, while $0.05<\mathrm{p}<0.10$ was considered to represent a non-significant, but clear trend in cell or tissue response. Values are reported as the mean \pm 
one standard deviation (SD). Brightness and contrast were adjusted equally across all of the images for improved visibility.

\section{Results}

\subsection{Effect of solution concentration on morphology of electrospun nanofibers}

According to previous work [22], the concentration of $8 \mathrm{wt} . \%$ was selected to prepare electrospun PCL nanofibers with diameters in the range from $200 \mathrm{~nm}$ to $1 \mu \mathrm{m}$. To prepare electrospun PCL/PLA nanofibers, different concentrations of 7, 8, 9, and 10 wt.\% were made; and morphologies of the resulting electrospun nanofibers were investigated. It was evident that the PCL/PLA solution with the concentration of $7 \mathrm{wt} . \%$ led to beaded nanofibers (Fig. 1A), and the diameters of these electrospun nanofibers were in the range of 120-900 $\mathrm{nm}$. The increase of PCL/PLA concentration to 8, 9, and $10 \mathrm{wt} \%$ (Figs. 1B, 1C, and 1D) led to the increase of nanofiber diameters to $250 \mathrm{~nm}-1 \mu \mathrm{m}, 400 \mathrm{~nm}-1.5 \mu \mathrm{m}$, and $500 \mathrm{~nm}-2 \mu \mathrm{m}$, respectively. These nanofibers had relatively uniform morphologies, and the mats contained very few beads and/or beaded-nanofibers. Hence, the solution with the concentration of 8 wt.\% was chosen to prepare electrospun PCL/PLA blend nanofibers. 

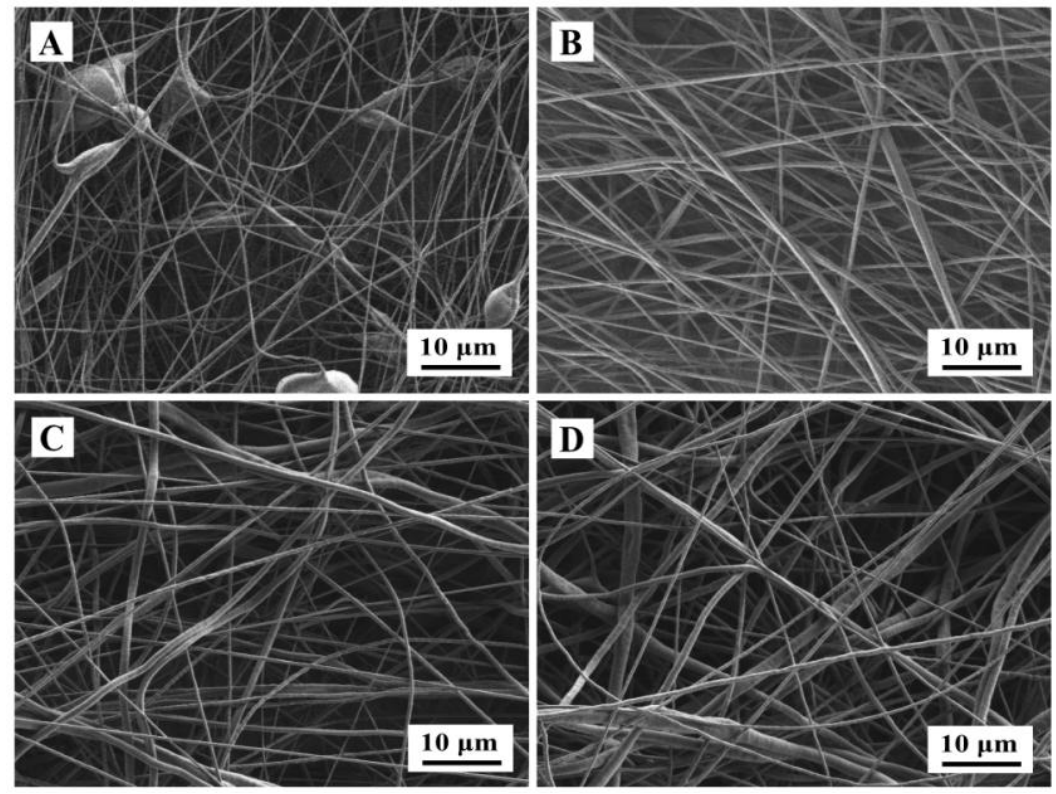

Fig. 1. SEM images showing the representative morphologies of PCL/PLA blend nanofibers electrospun from the solutions with PCL/PLA concentrations of (A) 7 wt.\%, (B) 8 wt.\%, (C) 9 wt.\%, and (D) $10 \mathrm{wt} . \%$, respectively.

\subsection{Morphologies and properties of the scaffolds}

As shown in Fig. 2A1 and 2B1, both as-electrospun PCL and PCL/PLA nanofibrous mats consisted of almost randomly overlaid nanofibers with diameters ranging from $200 \mathrm{~nm}$ to $1 \mu \mathrm{m}$ for PCL nanofibers, and from $150 \mathrm{~nm}$ to $2 \mu \mathrm{m}$ for PCL/PLA blend nanofibers. The morphologies of both nanofibers were relatively uniform, and both mats contained very few microscopically identifiable beads and/or beaded-nanofibers. Upon mechanical grinding, fragmented/tiny PCL nanofibrous mats/pieces (Fig. 2A2) as well as short individual PCL nanofibers (Fig. 2A3) were obtained. While for PCL/PLA blend, it appeared that only fragmented/tiny nanofibrous mats/pieces could be clearly identified (Fig. 2B1 \& B3). It is necessary to note that the nanofiber morphologies of both PCL and PCL/PLA blend could be 
well retained after grinding, dispersing, agglomerating, and the subsequent freeze drying (Fig. 2C1 to 2F3).

The parameters of different nanofibrous scaffolds are shown in Tables 1. It is evident that 3D electrospun nanofibrous scaffolds (i.e., 3D samples) have higher values of porosity while lower values of density, as compared with as-electrospun nanofibrous mats (i.e., 2D samples). The 3D samples had interconnected and hierarchically structured pores with sizes ranging from sub-micrometers to $\sim 300 \mu \mathrm{m}$, as shown in Figs. $2 \mathrm{C} 1$ to $2 \mathrm{~F} 3$, while for the $2 \mathrm{D}$ samples, the pore sizes were from sub-micrometers to several micrometers. It is known that the porosity and pore structure of a scaffold have significant impacts on cellular migration, proliferation, differentiation, as well as tissue formation; furthermore, cells and tissues could exhibit different responses to different pore sizes/structures [32, 33]. For example, in a 3D scaffold with hierarchically structured pores, the macropores with sizes in hundreds of micrometers would maintain the structural stability of scaffold, support cell proliferation, ECM deposition, and tissue formation; while the pores with sizes in tens of micrometers or smaller would facilitate the diffusion of nutrients and formation of vascularization. Moreover, research endeavors have also suggested that the pores with sizes in sub-micrometers might have important effects on controlling some cell behaviors such as attachment/seeding and gene expressions [34-36]. 

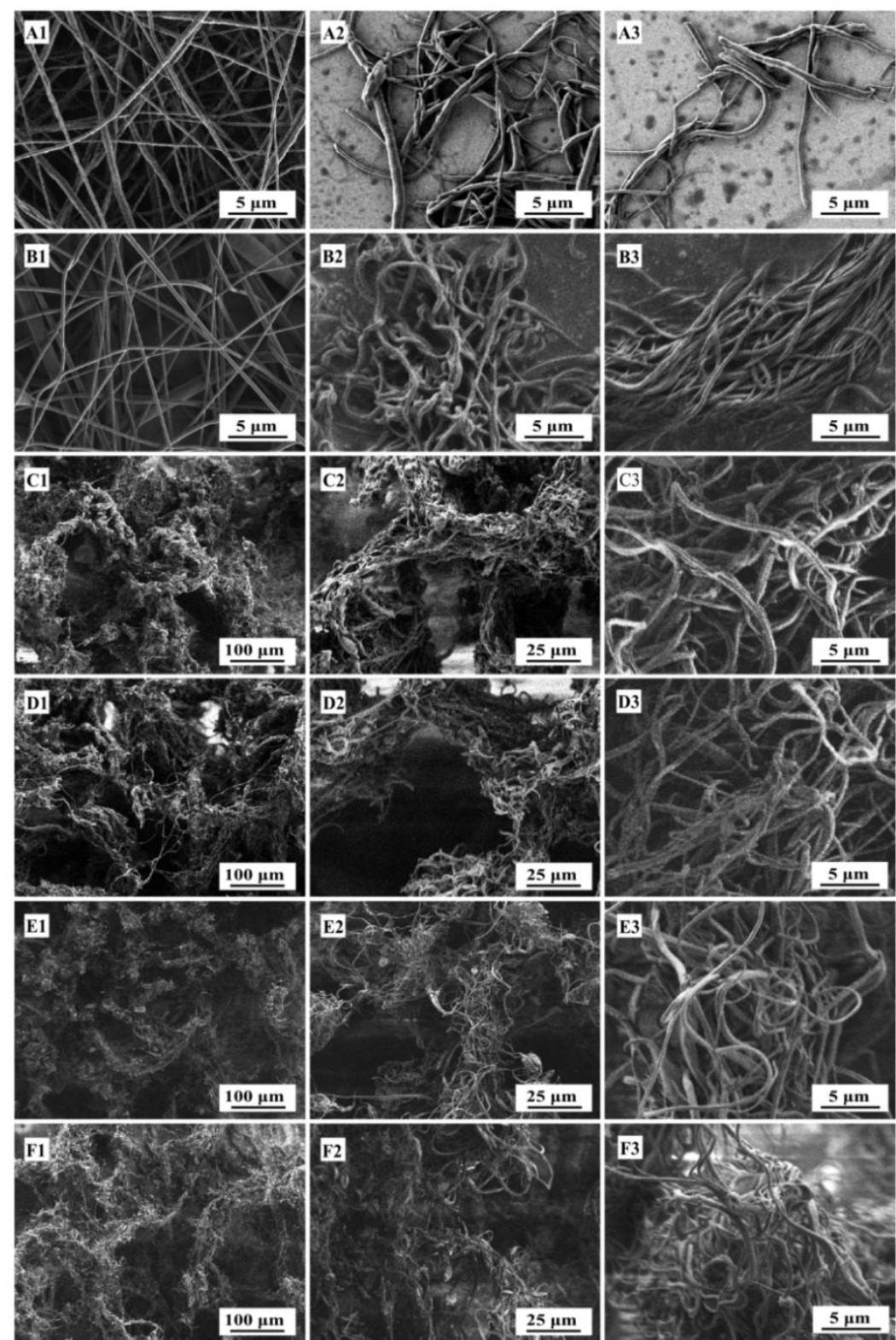

Fig. 2. SEM images showing the typical morphologies of (A1) as-electrospun PCL nanofibrous mat, (A2) fragmented/tiny PCL nanofibrous mat/piece, (A3) short individual PCL nanofibers, (B1) as-electrospun PCL/PLA nanofibrous mat, (B2 and B3) fragmented/tiny PCL/PLA nanofibrous mat/piece; $(\mathrm{C} 1, \mathrm{C} 2$, and C3) outer surface and (D1, D2, and D3) inner surface of 3D electrospun PCL nanofibrous scaffold; (E1, E2, and E3) outer surface and (F1, F2, and F3) inner surface of 3D electrospun PCL/PLA nanofibrous scaffold. 
Table 1.

Parameters of different nanofibrous scaffolds

\begin{tabular}{|c|c|c|c|c|c|c|c|c|c|}
\hline & & 1 & 2 & 3 & 4 & 5 & 6 & Mean & SD \\
\hline \multirow{4}{*}{$\begin{array}{l}\text { Porosity } \\
\qquad(\%)\end{array}$} & PCL-2D & 73.2 & 73.1 & 73.5 & 72.7 & 72.6 & 72.6 & 72.9 & 0.4 \\
\hline & PCL/PLA-2D & 77.4 & 77.3 & 77.3 & 77.0 & 77.0 & 76.9 & 77.2 & 0.2 \\
\hline & PCL-3D & 97.0 & 97.1 & 97.0 & 97.4 & 97.3 & 97.2 & 97.2 & 0.14 \\
\hline & PCL/PLA-3D & 96.0 & 96.2 & 95.9 & 95.9 & 95.5 & 95.3 & 95.8 & 0.3 \\
\hline \multirow{4}{*}{$\begin{array}{l}\text { Density } \\
\left(\mathrm{g} / \mathrm{cm}^{3}\right)\end{array}$} & PCL-2D & 0.307 & 0.307 & 0.303 & 0.312 & 0.312 & 0.313 & 0.309 & 0.003 \\
\hline & PCL/PLA-2D & 0.262 & 0.264 & 0.264 & 0.267 & 0.267 & 0.268 & 0.265 & 0.002 \\
\hline & PCL-3D & 0.033 & 0.031 & 0.032 & 0.028 & 0.030 & 0.031 & 0.030 & 0.001 \\
\hline & PCL/PLA-3D & 0.046 & 0.044 & 0.047 & 0.047 & 0.055 & 0.054 & 0.048 & 0.004 \\
\hline
\end{tabular}

\subsection{Mechanical properties of PCL-3D and PCL/PLA-3D scaffolds}

Fig. 3 depicts the stress-strain curves of PCL-3D and PCL/PLA-3D nanofibrous scaffolds. Both scaffolds exhibited typical behaviors of 3D nanofibrous scaffold undergoing deformation as described in literature [22]. The PCL/PLA-3D scaffolds had higher stress values compared to PCL-3D scaffolds at both dry and wet conditions. The general shapes of stress-strain curves for both types of scaffolds were similar; the PCL-3D scaffolds showed higher stress at wet condition than that at dry condition, while the PCL/PLA-3D scaffolds appeared to have similar stress-strain behaviors at both wet and dry conditions. During compressive tests, no fractured/shattered scaffolds were observed. These results indicated that 
the incorporation of $20 \mathrm{wt} . \%$ PLA into PCL would increase the stiffness/modulus while would not distinguishably vary the ductility/elasticity of the resulting PCL/PLA-3D scaffolds.
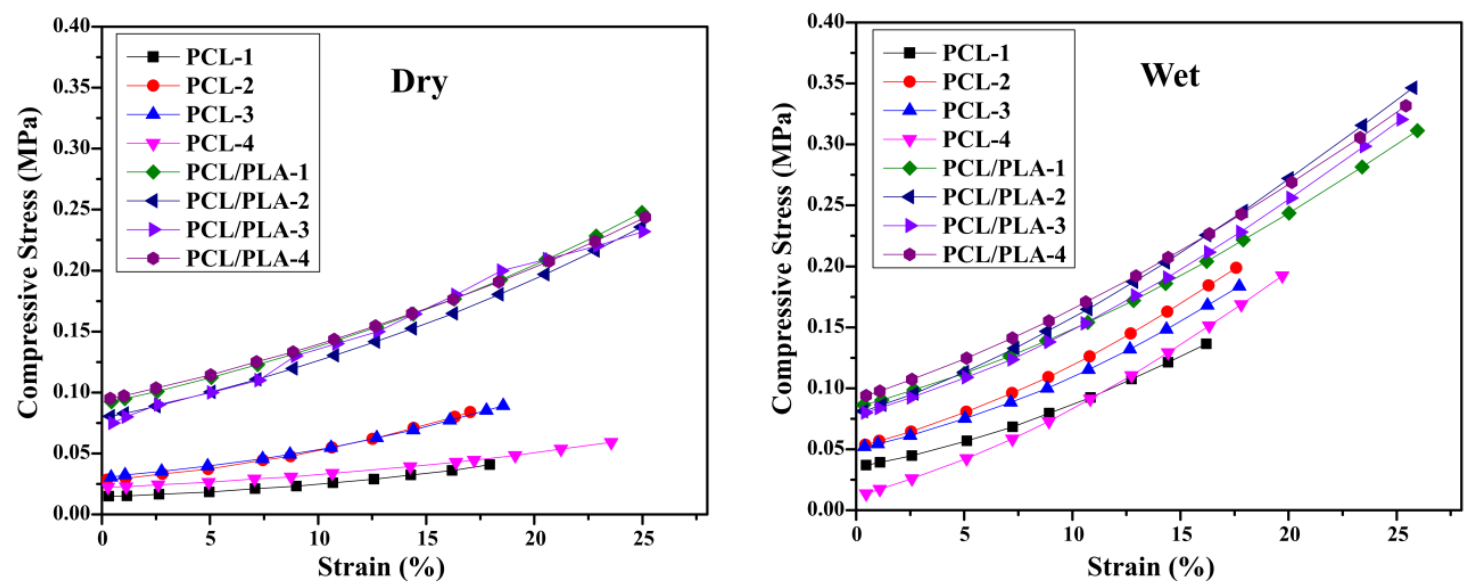

Fig. 3. Compressive stress-strain curves acquired from PCL-3D and PCL/PLA-3D scaffolds under both dry (left) and wet (right) conditions.

\subsection{Bioactivity of PCL-3D and PCL/PLA-3D scaffolds}

To study in vitro bioactivity of PCL-3D and PCL/PLA-3D scaffolds, the prepared scaffolds were immersed in SBF for different time periods. The formation of apatite-like deposits was noticeable on PCL/PLA scaffolds after 4-day immersion in SBF (Fig. 4A2), while few deposits could be identified on PCL scaffolds (Fig. 4A1). The apatite on PCL/PLA scaffolds developed into plate after 14 days of immersion (Fig. 4A6), while the apatite formed on PCL scaffolds exhibited graininess (Fig. 4A5), similar to the morphology observed on PCL/PLA scaffolds after 4-day immersion in SBF. These SEM results demonstrated that more and faster apatite could be formed on the PCL/PLA-3D scaffolds compared to that of PCL-3D scaffolds, suggesting that the incorporation of PLA into PCL was able to improve the bioactivity of the resulting PCL/PLA blend nanofiber. This result was further supported 
by the ATR data acquired from PCL-3D and PCL/PLA-3D scaffolds immersed in SBF (Fig. 5). After 4 days of SBF immersion, the characteristic peaks around 631 and $1650 \mathrm{~cm}^{-1}$, which could be attributed to the $\mathrm{OH}^{-}$groups in hydroxyapatite [37, 38], were detected on the PCL/PLA-3D scaffolds, whereas no such characteristic peaks were observed on the PCL-3D scaffolds.

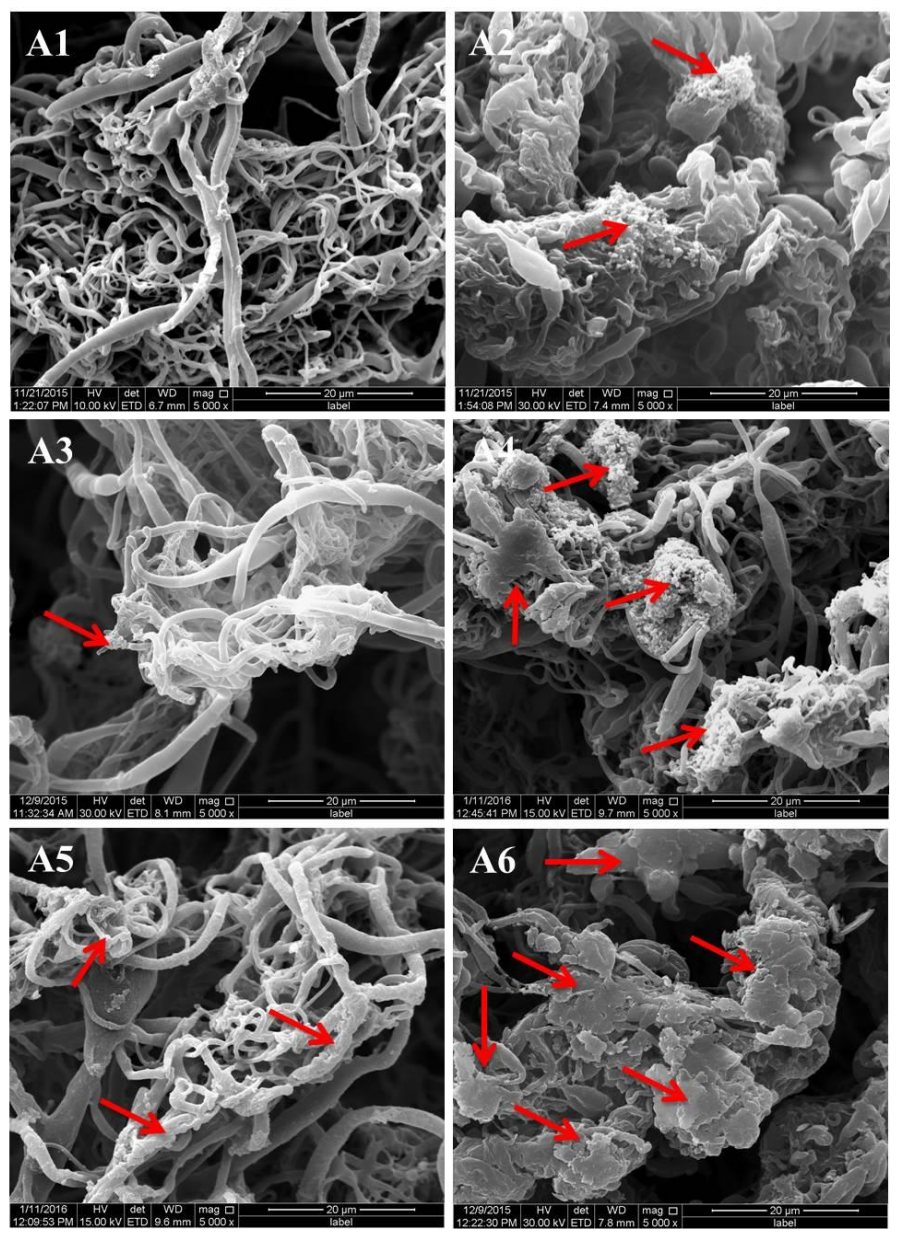

Fig. 4. SEM images of PCL-3D and PCL/PLA-3D scaffolds immersed in SBF after 1 day: (A1) PCL, (A2) PCL/PLA; after 7 days: (A3) PCL, (A4) PCL/PLA; and after 14 days: (A5) PCL, (A6) PCL/PLA. 

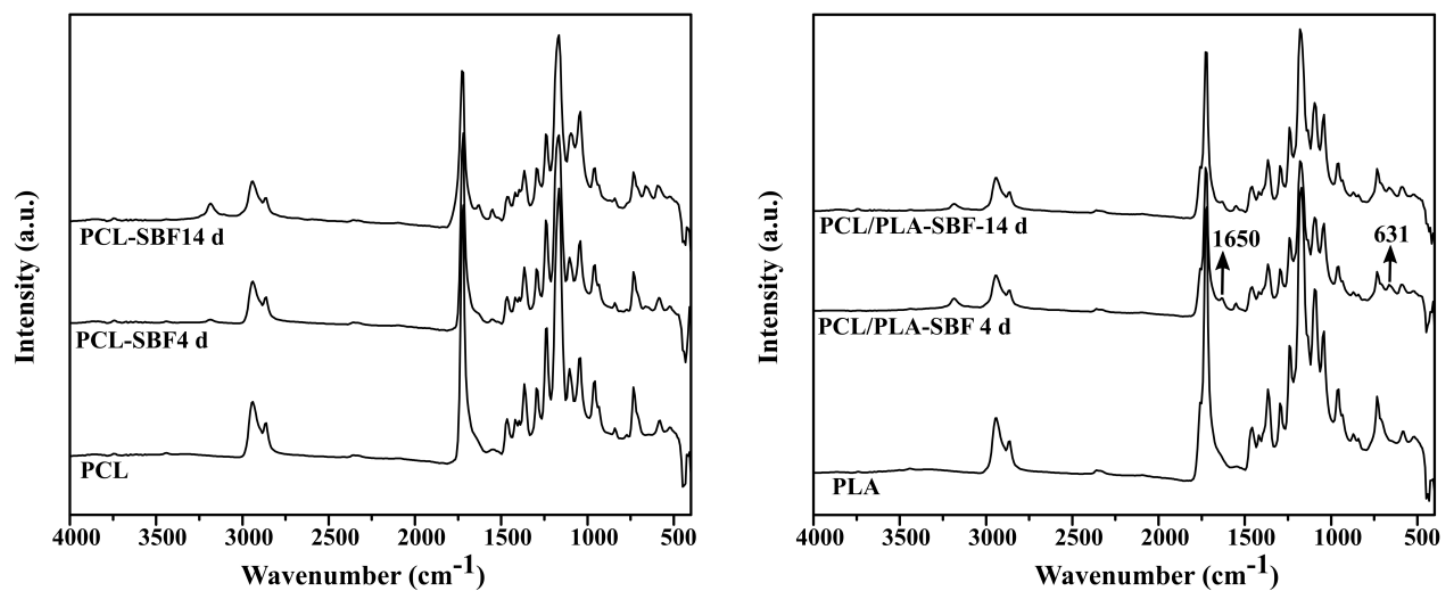

Fig. 5. ATR spectra acquired from PCL-3D (left) and PCL/PLA-3D (right) scaffolds after immersion in SBF for different days (i.e., 0, 4, and 14 days).

\subsection{Cell viability and morphology on PCL-3D and PCL/PLA-3D scaffolds}

Cell viabilities of hMSCs on PCL-3D and PCL/PLA-3D scaffolds were quantitatively measured by MTS assay after culturing for 1 and 3 days. As shown in Fig. 6A, both scaffolds exhibited similar cell viabilities after 1 day of culture. However, on day 3, the cell viability on PCL/PLA-3D scaffolds was moderately but significantly higher than that on PCL-3D scaffolds $(P<0.05)$. Consistent with the cell viability results, the cell proliferation results (Fig. S1.) indicated that hMSCs grew significantly faster on PCL/PLA-3D scaffolds than on PCL-3D scaffolds.

In addition to cell viabilities, hMSCs morphologies on PCL-3D and PCL/PLA-3D scaffolds were investigated after culturing for $16 \mathrm{~h}$. As shown in Fig. 6B1 and B2, cells adhered and spread well with a typical fibroblastic morphology on both types of scaffolds. No distinguishable differences were observed between the cell morphologies on the two types 
of scaffolds. These results suggested that the incorporation of PLA into PCL nanofiber slightly increased the cell viability while had little impact on cell morphology of hMSCs.
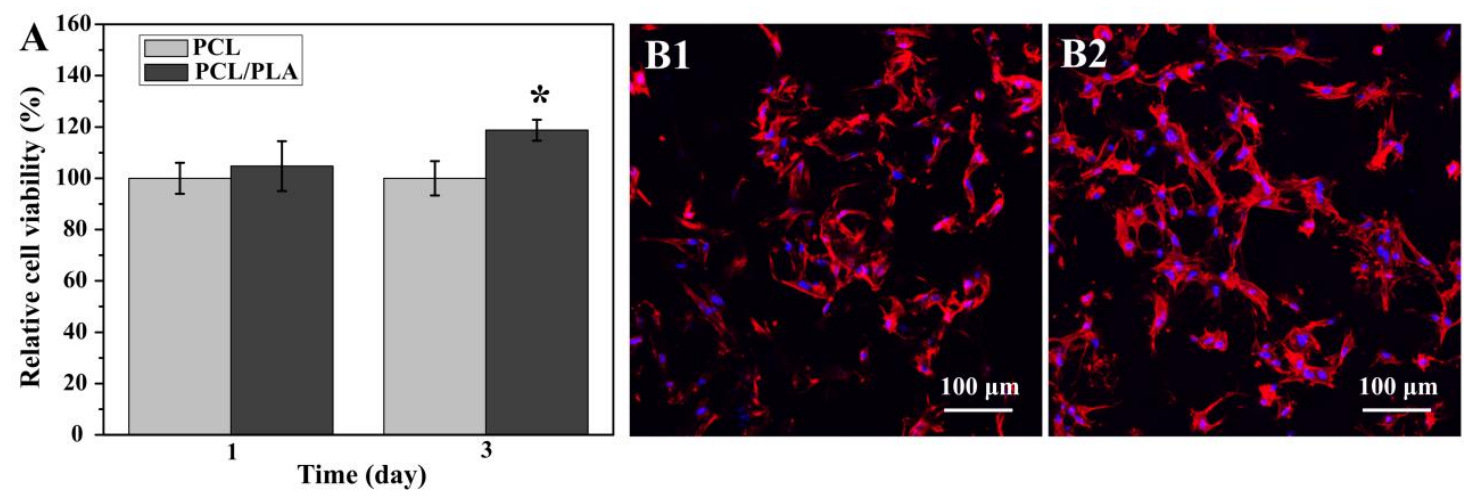

Fig. 6. (A) hMSCs viabilities on PCL-3D and PCL/PLA-3D scaffolds after culturing for 1 and 3 days. hMSCs morphologies on (B1) PCL-3D and (B2) PCL/PLA-3D scaffolds after culturing for $16 \mathrm{~h}$. Note that PCL-3D scaffolds are included as control samples. Data are expressed as mean $\pm \mathrm{SD}(n=3)$.

\subsection{Osteogenic differentiation of hMSCs on PCL-3D and PCL/PLA-3D scaffolds}

To study the effects of scaffolds on the osteogenic differentiation, hMSCs were cultured on two different 3D scaffolds in either growth medium (GM) or osteoinductive medium (OI). As the early osteogenic differentiation marker, ALP activity of hMSCs on 3D scaffolds were both qualitatively stained and quantitatively measured. Compared to GM, OI substantially elevated the ALP activity of hMSCs (red) cultured on both PCL-3D and PCL/PLA-3D scaffolds (Fig. S2.). Intriguingly, PCL/PLA-3D scaffolds were able to significantly increase the ALP activity $(P<0.05)$ in OI medium as compared to PCL-3D scaffolds (Fig. 7A). Additionally, the mineralization (formed at the late stage of osteogenic differentiation) was studied through analyzing the calcium content after 3-week culture. 
Although little calcium was detected on either type of scaffolds in GM medium, extensive amount of calcium was produced after cultured in OI medium for 3 weeks. Moreover, considerably more amount of calcium was detected from PCL/PLA-3D scaffolds than that from the PCL-3D scaffolds $(P<0.05)$. Consistent to the ALP activity and calcium content data, the gene expression results also indicated that hMSCs on PCL/PLA-3D scaffolds had significantly higher osteogenic differentiation, including both ALP and BSP (mature osteogenic marker gene), than hMSCs on PCL-3D scaffolds in OI condition. Similarly, no appreciable difference was observed in GM condition after 7 days of cell culture (Fig. 7C1 and $\mathrm{C} 2$ ). These results indicated that the incorporation of PLA into PCL (i.e., the PCL/PLA-3D scaffold) was able to significantly increase osteogenic differentiation of hMSCs in vitro. 

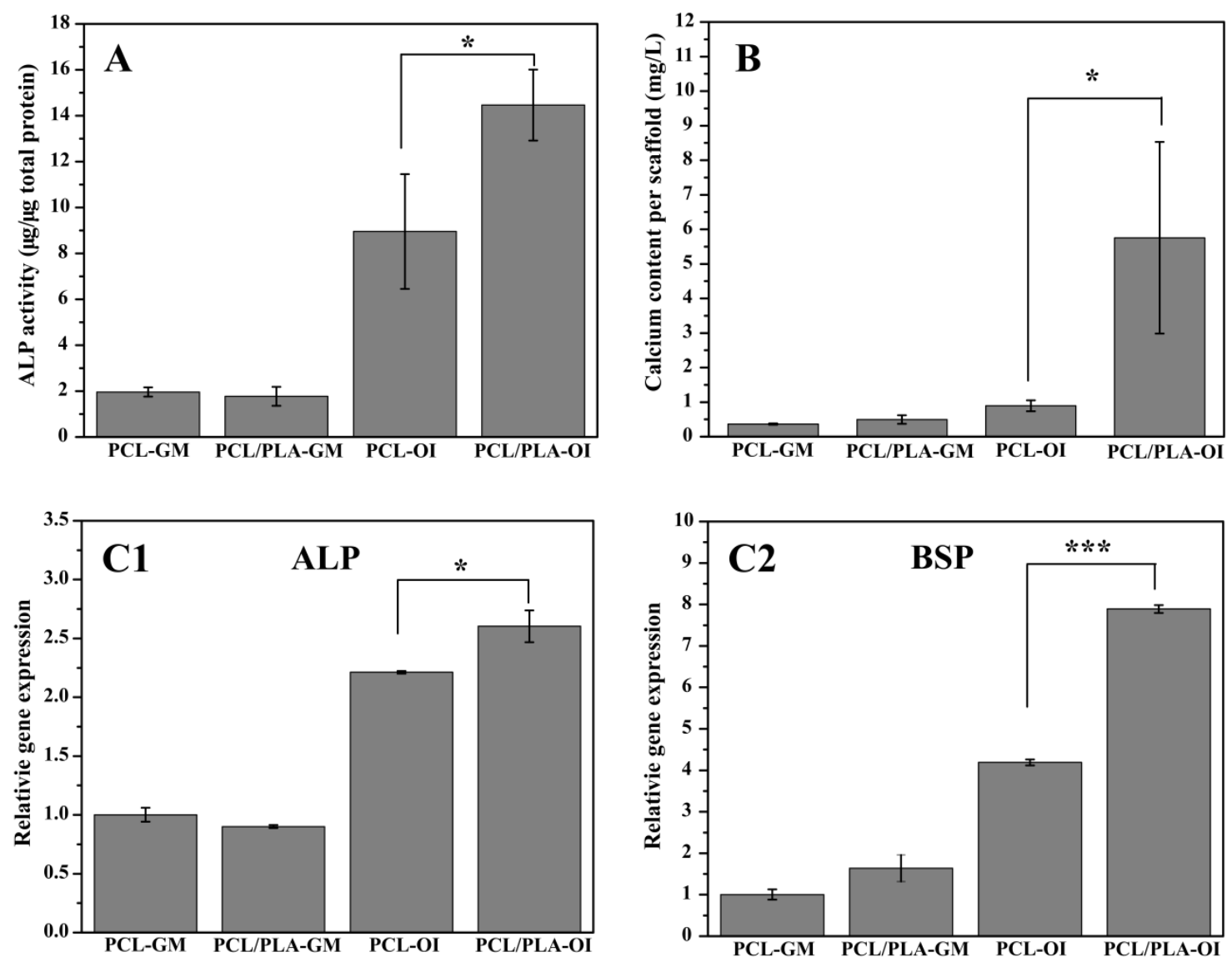

Fig. 7. ALP activity of hMSCs cultured on (A) PCL-3D and PCL/PLA-3D scaffolds for 10 days in growth medium (GM) and osteoinductive medium (OI). ALP activity was normalized by total protein content. (B) Calcium contents in PCL-3D and PCL/PLA-3D scaffolds were measured after culturing in $\mathrm{GM}$ and $\mathrm{OI}$ for 3 weeks. (C1 and $\mathrm{C} 2$ ) Osteogenic marker gene expressions (ALP and BSP) were studied by real-time PCR assay after 7 days of cell culture. Data are expressed as mean $\pm \mathrm{SD}(n=3) . P<0.05, * * P<0.01, * * * P<0.001$.

To study the potential contribution of chemical degrades from scaffolds on hMSCs functions, we collected the scaffold extracts from both PCL and PCL/PLA-3D scaffolds. Consistent to the results on scaffolds, PCL/PLA-3D scaffold extract was able to significantly increase the ALP activity $(P<0.05)$ of hMSCs when supplemented with OI medium as 
compared to PCL-3D scaffold extract (Fig. 8A). Intriguingly, the VEGF expression in hMSCs was significantly higher in PCL/PLA-3D scaffold extract than in PCL-3D scaffold extract; while OI medium largely reduced VEGF level in both scaffold extracts (Fig. 8B).
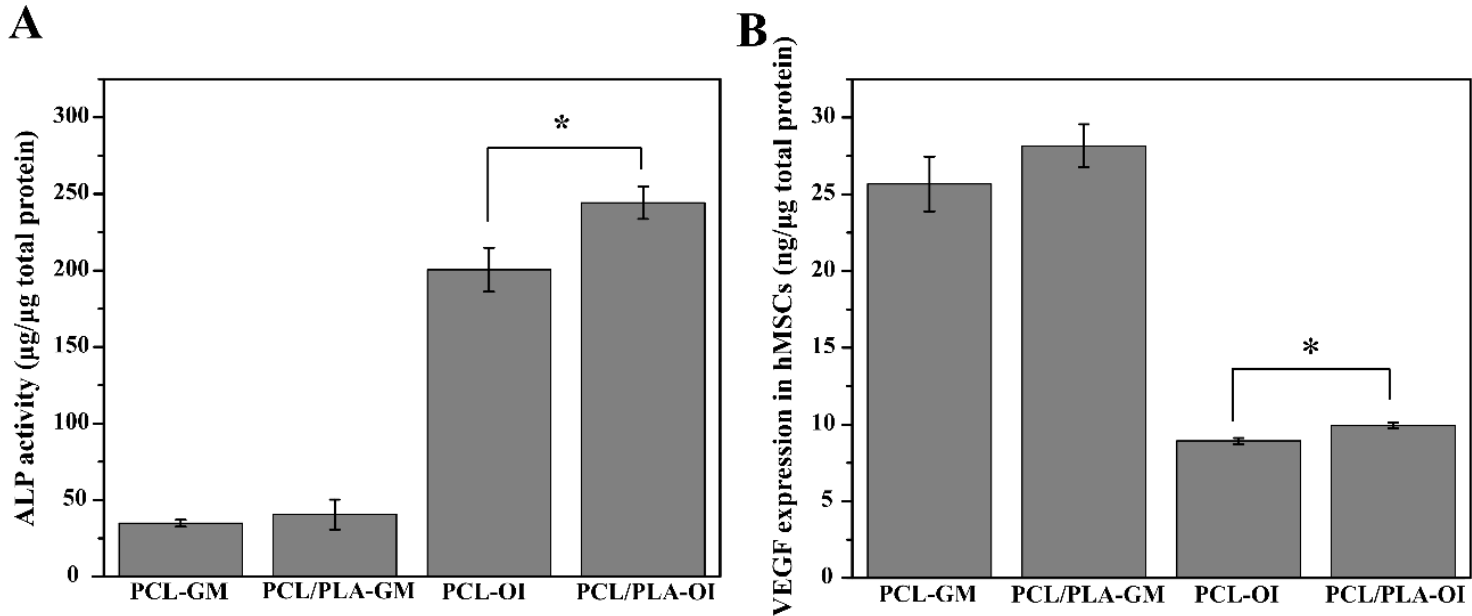

Fig. 8. (A) ALP activity of hMSCs cultured on PCL-3D and PCL/PLA-3D scaffold extracts, (B) expression of VEGF in hMSCs cultured in PCL-3D and PCL/PLA-3D scaffold extracts.

\subsection{Cranial bone regeneration on PCL-3D and PCL/PLA-3D scaffolds}

To study the influences of scaffolds on new bone formation in vivo, the PCL-3D and PCL/PLA-3D scaffolds (with or without rhBMP2) were implanted into mouse critical-sized cranial bone defects for up to 6 weeks. The radiographic examination results indicated that no significant increase of radiopacity in all of the implanted scaffolds even with low dose of rhBMP2 (left panel, Fig. 9). The macro-views of histologic slides were included to show the positions of the scaffolds, bone defects, and adjacent tissues (right panel, Fig. 9). Histological observation at low magnification (4X) revealed that abundant cells and tissues were all over the implanted scaffolds in all of the four groups, indicating the scaffolds with appropriate 
macropores and interconnected structures would support new cells/tissue growth (left panel, Fig. 10). At high magnification (20X), bone-like tissues were not observed in all of the five transplants of PCL group, while small new bone-like tissues were found in two out of six (2/6) transplants of PCL/PLA group. Note that these bony tissues were only formed in the areas which were close to the residual bones. Moreover, even supplemented with rhBMP2 into scaffolds, most of the PCL scaffolds (4/5) were still not able to form any bony tissue; while only one PCL scaffold was observed to form a little new bony tissue (1/5). On the other hand, all of the scaffolds from PCL/PLA-rhBMP2 group exhibited some new bony tissue formation (6/6) inside the scaffolds, even though the new bone was not able to bridge the critical defects and the boney structures were still immature without obvious marrow tissues. As shown in Table 2 , with the same amount of rhBMP2 addition $(0.75 \mu \mathrm{g} / \mathrm{scaffold})$, the average new bone area in PCL/PLA-rhBMP2 group (4.56\%) was considerably larger than that in PCL-rhBMP2 group (0.99\%). Hence, our in vivo results suggested that the newly developed PCL/PLA-3D scaffolds would provide a more favorable/desired microenvironment for mouse cranial bone formation as compared to the previously reported PCL-3D scaffolds. 


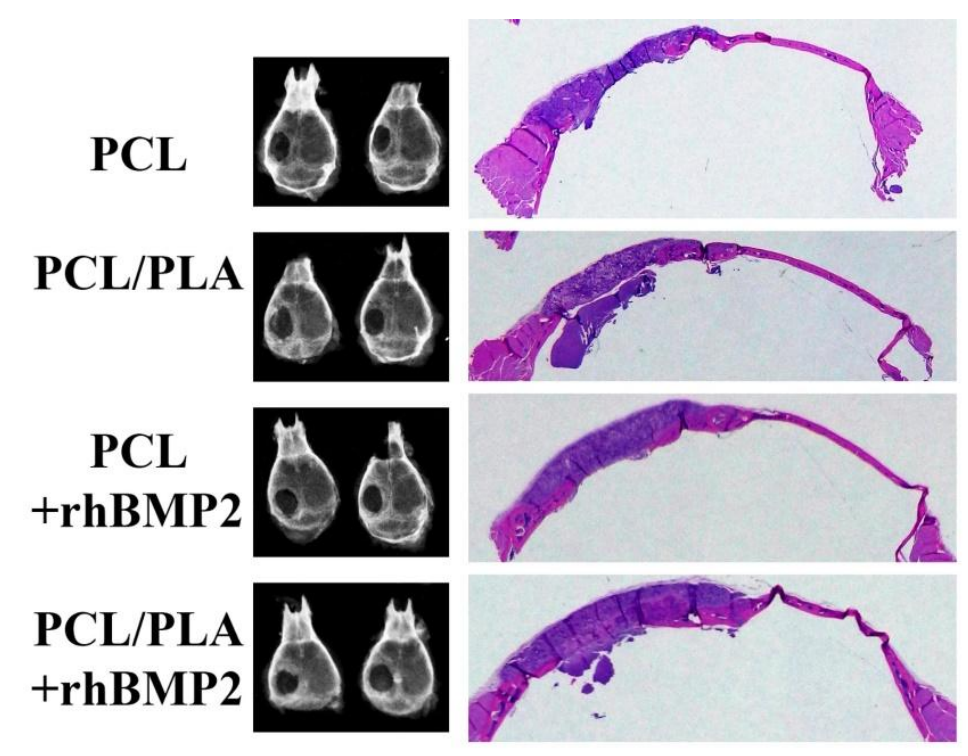

Fig. 9. Radiographic examination and macro-view of the histological scaffolds of PCL,

PCL/PLA, PCL-rhBMP2, and PCL/PAL-rhBMP2 groups after 6 weeks of implantation.

Representative data are shown $(n=5-6)$.

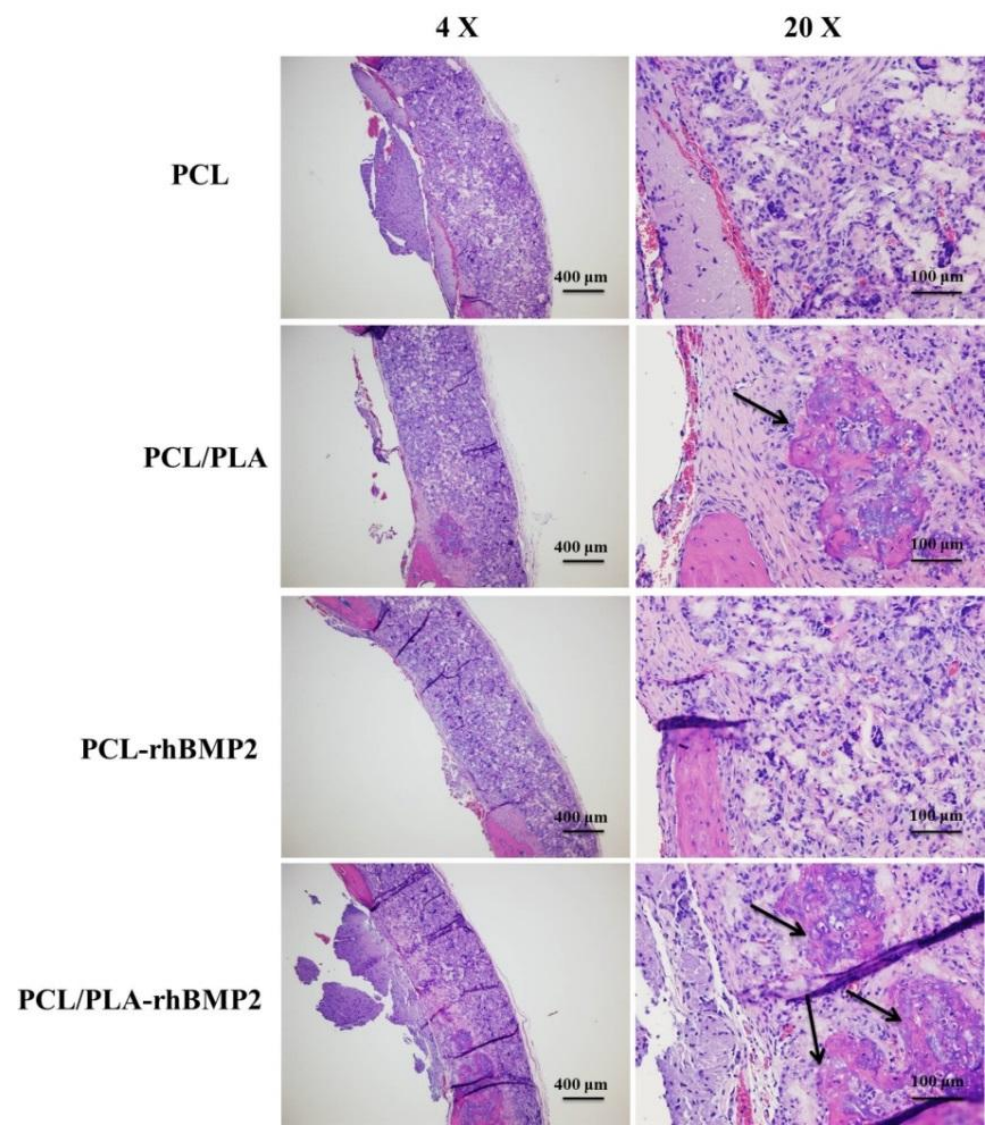

Fig. 10. H\&E staining of the repaired calvarias after 6 weeks of implantation in vivo. 
Table 2.

New bone formation parameters of PCL, PCL/PLA, PCL-rhBMP2, and PCL/PLA-rhBMP2 groups after 6 weeks of implantation in vivo.

\begin{tabular}{ccccc}
\hline & PCL & PCL/PLA & PCL-rhBMP2 & PCL/PLA-rhBMP2 \\
\hline New bone ratio & $0 / 5$ & $2 / 6$ & $1 / 5$ & $6 / 6$ \\
Average new bone area & & & & \\
percentage & $0 \%$ & $1.93 \%$ & $0.99 \%$ & $4.56 \%$ \\
\hline
\end{tabular}

\section{Discussion}

It has been a technological challenge to prepare 3D electrospun nanofibrous scaffolds with high porosity and interconnected macropores for tissue engineering applications [39]. To address this challenge, our group has recently developed an innovative TISA technique. In our recently reported work [22], the 3D electrospun PCL nanofibrous scaffolds with high porosity $(\sim 96 \%)$ and interconnected macropores (with sizes from tens to hundreds of micrometers) were explored; and these scaffolds were morphologically/structurally similar to natural ECM, thus well-suited for cell functions and tissue formation. To prepare more bone-formation favorable scaffolds, we incorporated a more bioactive polymer (i.e., PLA) into the relatively bio-inert PCL nanofibers; thus we prepared the 3D electrospun PCL/PLA blend nanofibrous scaffolds by using the TISA technique followed by the freeze drying method. These PCL/PLA-3D scaffolds possessed very similar macro/micro-porous structures to neat PCL-3D scaffolds; nevertheless, the compressive strength and in vitro mineralization capacity were substantially improved. Compared to neat PCL-3D scaffolds, the PCL/PLA-3D 
blend scaffolds not only enhanced hMSCs osteogenic differentiation in vitro, but also promoted cranial bone formation in vivo. As the two widely studied and FDA approved polymers, PCL and PLA can both be used to prepare 3D electrospun nanofibrous scaffolds, indicating that the TISA technique is an innovative and versatile approach for making tissue engineering scaffolds from as-electrospun nanofibrous mats.

"Bioactivity" is referring to the ability that a biomaterial can induce the formation of hydroxyapatite on its surface, when the biomaterial is immersed in SBF. This bioactivity test is widely adopted in vitro to assess whether the biomaterial is favorable for bone bonding and formation in vivo, since it has been reported that in vitro hydroxyapatite formation can be correlated to in vivo bioactivity of a biomaterial [40-42]. It has been reported that PCL scaffold is difficult to form hydroxyapatite probably due to the bio-inertness and slow degradation nature [8]. Thus the incorporation of more bioactive PLA (which is also able to degrade relatively fast) into PCL nanofibers would considerably improve the blend scaffold's bioactivity. The in vitro bioactivity study indicated that hydroxyapatite could be formed sooner and faster on PCL/PLA blend scaffolds compared to neat PCL scaffolds. In this work, PLA with 4.2\% D-lactide was used; and the amorphous D-lactide would be able to accelerate the degradation of PLA [43] and might promote the mineral formation. In addition to degradation, hydrophilicity change could also be a reason for the improved bioactivity [44]. It is necessary to note that no significant difference was detected between the water contact angles on PCL-2D and PCL/PLA-2D nanofibrous mats/scaffolds (data not shown), indicating that the PLA incorporation did not distinguishably vary the hydrophobicity of the resulting 
PCL/PLA blend nanofibrous mats. Furthermore, we did not find any significant changes in the microstructures between the two types of 3D scaffolds based on our experimental results. Therefore, we believe that the existence of amorphous D-lactide might be the main reason to facilitate the formation of hydroxyapatite. To study the detailed mechanism and further improve the mineralization ability of the $3 \mathrm{D}$ scaffolds, we plan to judiciously tailor the amount of amorphous D-lactide in the scaffolds in our future studies.

In addition to the studies of in vitro hydroxyapatite formation ability, our cell culture studies revealed that the incorporation of PLA was able to increase cell viability and osteogenic differentiation. Instead of mouse cells (which were used in our previous work [22]), hMSCs were used in current work because of the potential species differences [45]. We believe that using human stem cells would provide more clinically relevant information from our studies. It is well known that both PCL and PLA possess excellent biocompatibility, which was verified again by our in vitro cell viability study that no difference could be identified after 1 day of culture on both scaffolds. However, after 3 days, cells on PCL/PLA-3D scaffolds exhibited significantly higher cell viability than those on PCL-3D scaffolds. This might be attributed to the increased mechanical properties of PCL/PLA-3D scaffolds. Previous research endeavors suggested that the higher mechanical strength might potentially provide better mechanical support for cell growth by providing enough oxygen and nutrient exchanges, which is known as a major challenge for cell culture in thick 3D scaffolds [46, 47]. Additionally, it has been proven that the mechanical properties of ECM are capable of modulating cell behaviors, such as spread area, morphology, and gene 
expression profile. For example, it was reported that MSCs on the soft substrates had less spread, fewer stress fiber, and less proliferation rate than MSCs on stiff substrates [48-50]. In addition to cell viability, our in vitro results also indicated that PCL/PLA-3D scaffolds significantly promoted more potent osteogenic differentiation of hMSCs compared to PCL-3D scaffolds. Consistent with the increased calcium content, the BSP gene expression was found significantly higher in PCL/PLA-3D scaffold as well. Our finding could be explained by that BSP would act as nucleus for the first apatite crystal and regulate the bone matrix mineralization $[51,52]$. The improved osteogenic differentiation might be attributed to the increased stiffness, because higher stiffness of ECM has been known to direct MSCs towards the osteogenic differentiation pathway (rather than chondrogenic differentiation pathway) [50]. Furthermore, the degradation products of PLA might also contribute to the increased osteogenic differentiation according to the scaffold extract studies. These in vitro results, therefore, indicated that the incorporation of PLA into PCL (i.e., the PCL/PLA-3D scaffolds) could significantly improve the hMSCs cell viability and osteogenic differentiation because of both the increased mechanical properties and the different chemical compositions.

Consistent with in vitro results, our in vivo results also indicated that the newly developed PCL/PLA-3D scaffolds were more advantageous/favorable for bone formation in a clinically-relevant mouse critical-size cranial bone defect model. Bone formation is primarily determined by the features of transplanted scaffolds, including mechanical properties, degradation rate, structure, chemical components, and others $[30,53,54]$. It was revealed that small amount of new bone tissues were formed in the transplanted PCL/PLA-3D scaffolds, 
even without addition of exogenous rhBMP2. Furthermore, to demonstrate the difference on bone formation ability of scaffolds, we added relative low dose of rhBMP2 (0.75 $\mu \mathrm{g})$ into each scaffold based on our preliminary studies. At this dose of rhBMP2, we found that all of the transplanted PCL/PLA-3D scaffolds had significant amounts of newly formed bony tissues; whereas few new bony tissues were formed in PCL-3D scaffolds. This remarkable difference between PCL-3D and PCL/PLA-3D scaffolds suggested that the incorporation of PLA considerably improved the features of 3D scaffolds for bone formation. In addition to the improved mechanical properties and increased bioactivity, the scaffold extract studies revealed that the degradation products of PLA might also contribute to the enhanced osteogenic differentiation and bone formation on PCL/PLA-3D scaffolds. Lactate (i.e., the degradation product of PLA) is known for being able to stimulate collagen synthesis and new vessel formation $[55,56]$. Although the extract of scaffolds only moderately increased the ALP activity and VEGF expression in vitro (after culturing for 10 days), the benefits of the degradation products from PCL/PLA might contribute more significantly to bone formation in vivo due to the much longer time period (i.e., 6 weeks) and different microenvironment. In summary, our experimental results suggested that the newly developed 3D electrospun PCL/PLA blend nanofibrous scaffolds would provide more favorable/desired microenvironment for cranial bone formation (than the previously reported $3 \mathrm{D}$ electrospun neat PCL nanofibrous scaffolds), although the detailed mechanisms might still need to be further investigated. 


\section{Conclusions}

For the first time, 3D electrospun PCL/PLA blend (mass ratio: 4/1) nanofibrous scaffolds were developed by using the TISA technique followed by the freeze drying method. These scaffolds had the high porosity of $\sim 95.8 \%$ and possessed the interconnected and hierarchically structured pores with sizes from sub-micrometers to $\sim 300 \mu \mathrm{m}$; therefore, the morphological structures of such scaffolds would mimic those found in natural ECM. More importantly, the incorporation of PLA into PCL significantly increased the mechanical properties (e.g., compressive strength) and bioactivity of the resulting PCL/PLA-3D blend scaffolds. Compared to PCL-3D scaffolds, PCL/PLA-3D scaffolds were able to substantially improve the cell viability and osteogenic differentiation of hMSCs under in vitro conditions. Additionally, the PCL/PLA-3D scaffold extract was able to moderately but significantly increase both ALP activity and VEGF expression in hMSCs compared to the PCL-3D scaffold extract. These results suggested that both mechanical and chemical properties would contribute to the improved osteogenic differentiation of hMSCs on PCL/PLA-3D scaffolds. Moreover, the in vivo results indicated that PCL/PLA-3D scaffolds would considerably promote/facilitate new bone formation in a critical-sized cranial bone defect mouse model. Therefore, both in vitro and in vivo results indicated that our novel 3D electrospun PCL/PLA blend nanofibrous scaffolds would be more desired/favorable for hMSCs osteogenic differentiation and cranial bone formation than the previously reported $3 \mathrm{D}$ electrospun (neat) PCL nanofibrous scaffolds. 


\section{Acknowledgements}

This work was supported by the EPSCoR program of National Science Foundation (Award No.: IIA-1335423) and by the Competitive Research Grant program of South Dakota Board of Regents (Award No.: UP1500172). The authors would like to acknowledge the assistance provided by the Sanford Research Imaging Core and Molecular Pathology Core, which were supported by the COBRE grants of National Institutes of Health (Grant No.: P20 GM103620 and P20 GM103548). The authors would also thank Dr. Erin B. Harmon for his outstanding technical assistance. Additionally, Jaqueline G.L. Cosme and Paulo H.S. Picciani would like to acknowledge the support from the CAPES Foundation (Science without Borders project) of the Ministry of Education of Brazil (Grant No.: 99999.010045/2014-07). 


\section{References}

[1] Cabraja M, Kroppenstedt S. Bone grafting and substitutes in spine surgery. J Neurosurg Sci 2012;56:87-95.

[2] Ng MH, Duski S, Tan KK, Yusof MR, Low KC, Mohamed Rose I, et al. Repair of segmental load-bearing bone defect by autologous mesenchymal stem cells and plasma-derived fibrin impregnated ceramic block results in early recovery of limb function. Biomed Res Int 2014;345910.

[3] Morelli S, Salerno S, Holopainen J, Ritala M, De Bartolo L. Osteogenic and osteoclastogenic differentiation of co-cultured cells in polylactic acid-nanohydroxyapatite fiber scaffolds. J Biotechnol 2015;204:53-62.

[4] Heymer A, Bradica G, Eulert J, Nöth U. Multiphasic collagen fibre-PLA composites seeded with human mesenchymal stem cells for osteochondral defect repair: an in vitro study. J Tissue Eng Regen Med 2009;3:389-97.

[5] Khan Y, Yaszemski MJ, Mikos AG, Laurencin CT. Tissue engineering of bone: material and matrix considerations. J Bone Joint Surg 2008;90:36-42.

[6] Bose S, Roy M, Bandyopadhyay A. Recent advances in bone tissue engineering scaffolds. Trends Biotechnol 2012;30:546-54.

[7] Yao Q, Li W, Yu S, Ma L, Jin D, Boccaccini AR, et al. Multifunctional chitosan/polyvinyl pyrrolidone/45S5 Bioglass $^{\circledR}$ scaffolds for MC3T3-E1 cell stimulation and drug release. Mater Sci Eng C Mater Biol Appl 2015;56:473-80.

[8] Abedalwafa M, Wang F, Wang L, Li C. Biodegradable poly-epsilon-caprolactone 
(PCL) for tissue engineering applications: a review. Rev Adv Mater Sci 2013;34:123-40.

[9] Dhandayuthapani B, Yoshida Y, Maekawa T, Kumar DS. Polymeric scaffolds in tissue engineering application: a review. Inter J Polymer Sci 2011;290602.

[10] Ou K-L, Hosseinkhani H. Development of 3D in vitro technology for medical applications. Inter J Mol Sci 2014;15:17938-62.

[11] Wang J, Valmikinathan CM, Liu W, Laurencin CT, Yu X. Spiral-structured, nanofibrous, 3D scaffolds for bone tissue engineering. J Biomed Mater Res A 2010;93:753-62.

[12] Holzwarth JM, Ma PX. Biomimetic nanofibrous scaffolds for bone tissue engineering. Biomaterials 2011;32:9622-9.

[13] Liu X, Jin X, Ma PX. Nanofibrous hollow microspheres self-assembled from star-shaped polymers as injectable cell carriers for knee repair. Nat Mater 2011;10:398-406.

[14] Wade RJ, Burdick JA. Engineering ECM signals into biomaterials. Mater Today 2012;15:454-9.

[15] Matson JB, Zha RH, Stupp SI. Peptide self-assembly for crafting functional biological materials. Curr Opin Solid State Mater Sci 2011;15:225-35.

[16] Wang X, Ding B, Li B. Biomimetic electrospun nanofibrous structures for tissue engineering. Mater Today 2013;16:229-41.

[17] Li WJ, Laurencin CT, Caterson EJ, Tuan RS, Ko FK. Electrospun nanofibrous 
structure: a novel scaffold for tissue engineering. J Biomed Mater Res 2002;60:613-21.

[18] Bhardwaj N, Kundu SC. Electrospinning: a fascinating fiber fabrication technique. Biotechnol Adv 2010;28:325-47.

[19] Blakeney BA, Tambralli A, Anderson JM, Andukuri A, Lim DJ, Dean DR, et al. Cell infiltration and growth in a low density, uncompressed three-dimensional electrospun nanofibrous scaffold. Biomaterials 2011;32:1583-90.

[20] Liu XH, Ma PX. Phase separation, pore structure, and properties of nanofibrous gelatin scaffolds. Biomaterials 2009; 30:4094-4103.

[21] $\mathrm{Hu} \mathrm{J}$, Liu XH, Ma PX. Induction of osteoblast differentiation phenotype on poly(L-lactic acid) nanofibrous matrix. Biomaterial 2008;29:3815-21.

[22] $\mathrm{Xu} \mathrm{T}$, Miszuk JM, Zhao Y, Sun H, Fong H. Electrospun polycaprolactone 3D nanofibrous scaffold with interconnected and hierarchically structured pores for bone tissue engineering. Adv Healthc Mater 2015;4:2238-46.

[23] Chen BY, Jing X, Mi HY, Zhao H, Zhang WH, Peng XF, et al. Fabrication of polylactic acid/polyethylene glycol (PLA/PEG) porous scaffold by supercritical $\mathrm{CO}_{2}$ foaming and particle leaching. Polym Eng Sci 2015;55:1339-48.

[24] Middleton JC, Tipton AJ. Synthetic biodegradable polymers as orthopedic devices. Biomaterials 2000;21:2335-46.

[25] Zhang G, Fiore GL, St. Clair TL, Fraser CL. Difluoroboron dibenzoylmethane PCL-PLA block copolymers: Matrix effects on room temperature phosphorescence. 
Macromolecules 2009;42:3162-9.

[26] Sun M, Downes S. Physicochemical characterisation of novel ultra-thin biodegradable scaffolds for peripheral nerve repair. J Mate Sci Mater Med 2009;20:1181-92.

[27] Sun M, Kingham PJ, Reid AJ, Armstrong SJ, Terenghi G, Downes S. In vitro and in vivo testing of novel ultrathin PCL and PCL/PLA blend films as peripheral nerve conduit. J Biomed Mater Res A 2010;93:1470-81.

[28] Kokubo T. Bioactive glass ceramics: properties and applications. Biomaterials $1991 ; 12: 155-63$.

[29] Sun H, Feng K, Hu J, Soker S, Atala A, Ma PX. Osteogenic differentiation of human amniotic fluid-derived stem cells induced by bone morphogenetic protein-7 and enhanced by nanofibrous scaffolds. Biomaterials 2010;31:1133-9.

[30] Sun H, Zhu F, Hu Q, Krebsbach PH. Controlling stem cell-mediated bone regeneration through tailored mechanical properties of collagen scaffolds. Biomaterials 2014;35:1176-84.

[31] Sun H, Jung Y, Shiozawa Y, Taichman RS, Krebsbach PH. Erythropoietin modulates the structure of bone morphogenetic protein 2-engineered cranial bone. Tissue Eng A 2012;18:2095-105.

[32] Karageorgiou V, Kaplan D. Porosity of 3D biomaterial scaffolds and osteogenesis. Biomaterials 2005;26:5474-91.

[33] Zeltinger J, Sherwood JK, Graham DA, Mueller R, Griffith LG. Effect of pore size and void fraction on cellular adhesion, proliferation, and matrix deposition. Tissue 
Eng 2001;7:557-72.

[34] Chiu LL, Montgomery M, Liang Y, Liu H, Radisic M. Perfusable branching microvessel bed for vascularization of engineered tissues. Proc NatI Acad Sci U S A 2012;109:E3414-23.

[35] Smith LA, Liu X, Hu J, Ma PX. The influence of three-dimensional nanofibrous scaffolds on the osteogenic differentiation of embryonic stem cells. Biomaterials 2009;30:2516-22.

[36] Liu J, Yue Z, Fong H. Continuous nanoscale carbon fibers with superior mechanical strength. Small 2009;5:536-42.

[37] Yao Q, Yang Y, Pu X, Yang L, Hou Z, Dong Y, et al. Preparation, characterization and osteoblastic activity of chitosan/polycaprolactone/in situ hydroxyapatite scaffolds. J Biomater Sci Polym Ed 2012;23:1755-70.

[38] Raynaud S, Champion E, Bernache-Assollant D, Thomas P. Calcium phosphate apatites with variable $\mathrm{Ca} / \mathrm{P}$ atomic ratio I. Synthesis, characterisation and thermal stability of powders. Biomaterials 2002;23:1065-72.

[39] Zhong SP, Zhang YZ, Lim CT. Fabrication of large pores in electrospun nanofibrous scaffolds for cellular infiltration: a review. Tissue Eng Part B 2012;18:77-87.

[40] Kokubo T, Takadama H. How useful is SBF in predicting in vivo bone bioactivity? Biomaterials 2006;27:2907-15.

[41] Rezwan K, Chen QZ, Blaker JJ, Boccaccini AR. Biodegradable and bioactive porous polymer/inorganic composite scaffolds for bone tissue engineering. Biomaterials 
2006;27:3413-31.

[42] Verné E, Bruno M, Miola M, Maina G, Bianco C, Cochis A, Rimondini L. Composite bone cements loaded with a bioactive and ferrimagnetic glass-ceramic: Leaching, bioactivity and cytocompatibility. Mater Sci Eng C Mater Biol Appl. 2015;53:95-103.

[43] Chen C-C, Chueh J-Y, Huang H-M, Lee S-Y. Preparation and characterization of biodegradable PLA polymeric blends. Biomaterials 2003;24:1167-73.

[44] Ma ZW, Mao ZW, Gao CY. Surface modification and property analysis of biomedical polymers used for tissue engineering. Colloid Surface B 2007;60:137-57.

[45] Czekanska EM, Stoddart MJ, Richards RG, Hayes JS. In search of an osteoblast cell model for in vitro research. Eur Cell Mater 2012;24:1-17.

[46] Loh QL, Choong C. Three-dimensional scaffolds for tissue engineering applications: role of porosity and pore size. Tissue Eng Part B Rev 2013;19:485-502.

[47] Gardel LS, Serra LA, Reis RL, Gomes ME. Use of perfusion bioreactors and large animal models for long bone tissue engineering. Tissue Eng Part B Rev 2013;20:126-46.

[48] Mullen CA, Vaughan TJ, Billiar KL, McNamara LM. The effect of substrate stiffness, thickness, and cross-linking density on osteogenic cell behavior. Biophys J 2015;108:1604-12.

[49] Park JS, Chu JS, Tsou AD, Diop R, Tang Z, Wang A, et al. The effect of matrix stiffness on the differentiation of mesenchymal stem cells in response to TGF- $\beta$. Biomaterials 2011;32:3921-30. 
[50] Lv H, Li L, Sun M, Zhang Y, Chen L, Rong Y, et al. Mechanism of regulation of stem cell differentiation by matrix stiffness. Stem Cell Res Ther 2015;6:103.

[51] Hunter GK, Goldberg HA. Modulation of crystal-formation by bone phosphoproteins - role of glutamic acid-rich sequences in the nucleation of hydroxyapatite by bone sialoprotein. Biochem J 1994;302:175-9.

[52] Ogata Y. Bone sialoprotein and its transcriptional regulatory mechanism. J Periodontal Res 2008;43:127-35.

[53] Yang W, Both SK, van Osch G, Wang Y, Jansen J, Yang F. Performance of different three-dimensional scaffolds for in vivo endochondral bone generation. Eur Cell Mater $2014 ; 27: 350-64$.

[54] Huebsch N, Lippens E, Lee K, Mehta M, Koshy ST, Darnell MC, et al. Matrix elasticity of void-forming hydrogels controls transplanted-stem-cell-mediated bone formation. Nat Mater 2015;14:1269-77.

[55] Trabold O, Wagner S, Wicke C, Scheuenstuhl H, Hussain MZ, Rosen N, et al. Lactate and oxygen constitute a fundamental regulatory mechanism in wound healing. Wound Repair Regen 2003;11:504-9.

[56] Schneider CC, Ateschrang A, Konigsrainer I, Glatzle J, Buhler S, Schaefer R, et al. Lactate influences the gene expression profile of human mesenchymal stem cells (hMSC) in a dose dependant manner. Cell Physiol Biochem 2012;30:1547-56. 


\section{Graphical abstract}

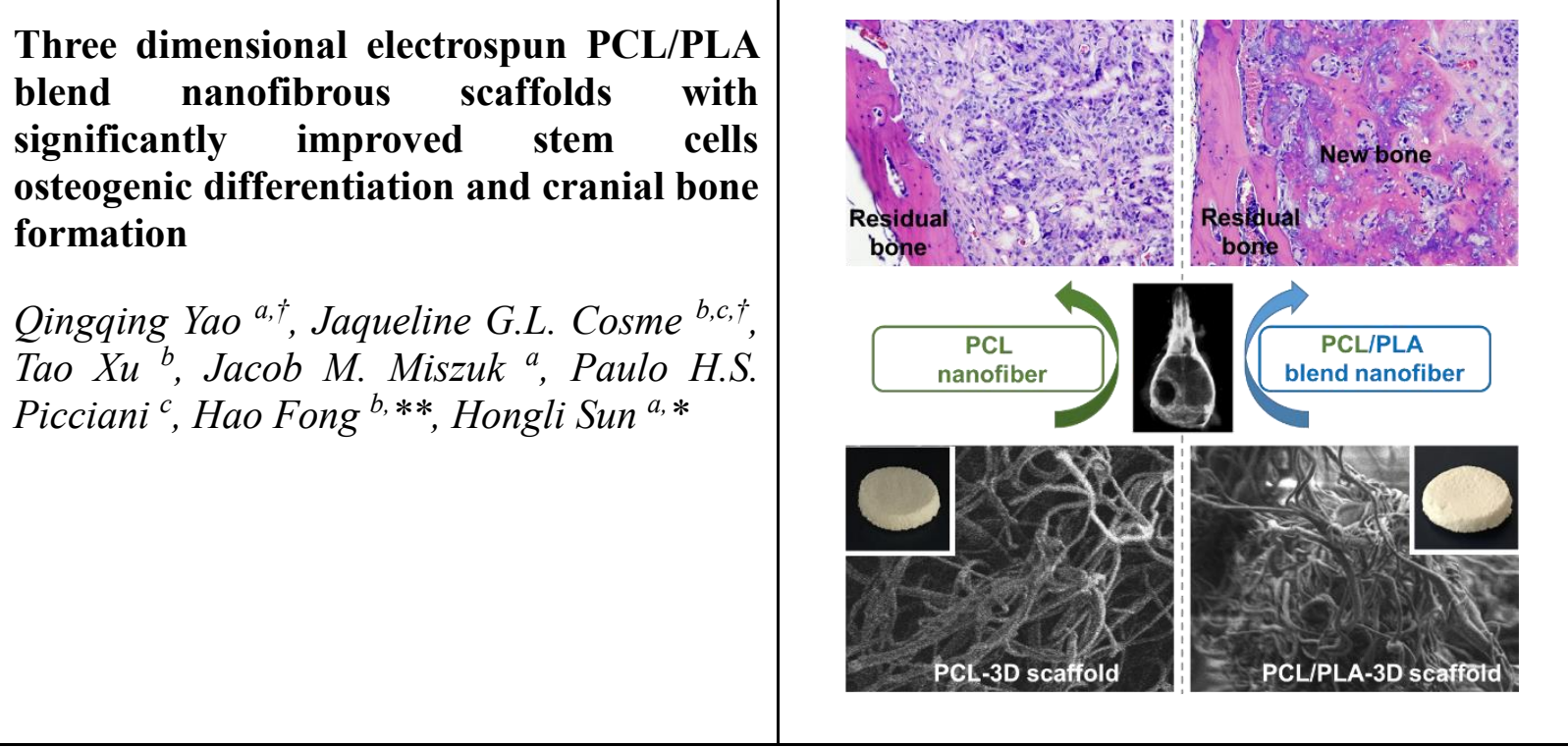

